\title{
A CHARACTERIZATION OF CONVEX HYPERSURFACES IN HADAMARD MANIFOLDS
}

\author{
MEHMET ERDOĞAN and GÜLŞEN YILMAZ
}

\begin{abstract}
The aim of this paper is to give a characterization of strictly convex hypersurfaces in a Hadamard manifold.
\end{abstract}

\section{Introduction}

Convex hypersurfaces in any space have interesting properties. Metrics of curvature bounded below arose initially in papers by A. D. Alexandrov as nonnegatively curved metrics on two-dimensional surfaces [3] and he also studied convex surfaces in Lobachevsky space [2]. After that Burago and Zalgaller gave some results about convex sets in Riemannian manifolds [8], [9]. On the other hand, it is a natural question to ask for the relations between curvature properties of the metric and the geometrical shape of the hypersurface. This question is especially interesting for the hypersurfaces of negatively curved spaces. A very nice paper had been written for locally convex hypersurfaces of negatively curved spaces by S. B. Alexander [1], and after him many others appeared. Most beautiful papers which have been written on this matter in the last decade belong to A. A. Borisenko [6], [7].

A well-known theorem due to Hadamard [13] states that if the second fundamental form of a compact immersed hypersurface $M$ of Euclidean space $E^{n+1}(n \geq 2)$ is positive definite, then $M$ is imbedded as the boundary of a convex body. Hadamard's theorem also implies that the following statements are equivalent:

(i) The second fundamental form $\sigma$ of $M$ is definite everywhere on $M$.

(ii) $M$ is orientable and the spherical map of Gauss $M \rightarrow S^{n}$ is a diffeomorphism.

(iii) The Gaussian curvature of $M$ never vanishes on $M$.

Received July 26, 2004. 
In the present paper, we will extend the results above to hypersurfaces in a Hadamard manifold $N$. In fact, we will prove

THEOREM. For a connected compact hypersurface $M$ in an $(n+1)$ dimensional Hadamard manifold $N(n \geq 2)$ the following conditions are equivalent :

(i) The second fundamental form $\sigma$ of $M$ is definite everywhere on $M$.

(ii) If the sectional curvatures of $N$ satisfy $K \leq-k<0$, then for a suitably chosen continuous unit normal $\zeta$ of $M$, the eigenvalues of the second fundamental form $\sigma$ satisfy $\lambda>-\sqrt{k}$ and $\lambda \neq 0$.

(iii) If $M$ is a compact orientable immersed hypersurface of a Hadamard manifold $N$, then $M$ is locally convex and homeomorphic to the sphere.

Moreover, any of the conditions above implies that $M$ is a strictly convex hypersurface in $N$.

\section{Preliminaries}

Let $M$ be an $n$-dimensional hypersurface in an $(n+1)$-dimensional Hadamard manifold $N$, that is, $M$ is an $n$-dimensional Riemannian submanifold embedded in $N$ with the induced Riemannian structure. We will denote by $g$ (resp. $\tilde{g}$ ) the Riemannian metric tensor of $M$ (resp. $N$ ). Let $T_{x} M$ be the tangent space to $M$ at $x$ and $T_{x} M^{\perp}$ be the normal space to $M$ at $x$. We denote by $\nabla$ (resp. $\tilde{\nabla}$ ) the covariant differentiation on $M$ (resp. $N$ ). Then, for tangent vector fields $X$, $Y$ and the unit normal field $\zeta$ on $M$, as is well known, the formulas of Gauss and Weingarten are

$$
\begin{aligned}
\tilde{\nabla}_{X} Y & =\nabla_{X} Y+\sigma(X, Y), \\
\tilde{\nabla}_{X} \zeta & =-A(X),
\end{aligned}
$$

where $\sigma$ is the second fundamental form of $M$ and satisfies

$$
\sigma(X, Y)=\sigma(Y, X)
$$

and $A$ is the symmetric linear transformation on each tangent space to $M$, which is called the shape operator.

Since $M$ is a hypersurface we may write

$$
\sigma(X, Y)=h(X, Y) \zeta
$$

Then, we easily see that

$$
h(X, Y)=\tilde{g}(\sigma(X, Y), \zeta)=g(A(X), Y) .
$$


The eigenvalues $\lambda_{1}, \lambda_{2}, \ldots, \lambda_{n}$ of the shape operator $A$ are called principal curvatures of $M$, and an orthonormal basis $e_{1}, e_{2}, \ldots, e_{n}$ such that

$$
A e_{i}=\lambda_{i} e_{i}, \quad 1 \leq i \leq n,
$$

are called principal vectors on $M$. In this case,

$$
\lambda_{i}=h\left(e_{i}, e_{i}\right), \quad i=1,2, \ldots, n .
$$

Furthermore,

$$
K_{n}=\lambda_{1} \lambda_{2} \ldots \lambda_{n}
$$

is called the Gaussian curvature of $M$

The second fundamental form $\sigma$ is definite at $x \in M$ if $\sigma(X, X) \neq 0$ for all nonzero vectors $X \in T_{x} M$, that is, $h$ is either positive definite or negative definite. $\sigma$ is said to be non-degenerate at $x$ if $h$ is non-degenerate at $x$.

We say that two unit geodesic

$$
\gamma_{i}: \mathrm{R} \rightarrow N, \quad i=1,2,
$$

are asymptotic if

$$
d\left(\gamma_{1}(t), \gamma_{2}(t)\right) \leq c, \quad \forall t \in \mathbf{R},
$$

for some constant $c \in \mathrm{R}$. The equivalence classes of this relation are called points at infinity and are denoted by $N(\infty)$. Also, we will denote by

$$
\gamma(\infty) \in N(\infty)
$$

the corresponding class for a geodesic $\gamma$. As is well known, for two points $p \in N$ and $z \in N(\infty)$, there exists a unique unit speed geodesic $\gamma$ with $\gamma(0)=p$ and $\gamma(\infty)=z$. A horoball in a Hadamard manifold $N$ is the domain obtained as the limit of the balls with their centers in a geodesic ray going to infinity and their corresponding geodesic spheres containing a fixed point. Namely, for a unit speed geodesic ray $\gamma$ the union of balls

$$
\bigcup_{t>0} B_{t}(\gamma(t))
$$

is the horoball $\operatorname{HB}(p, z)$ with center $\gamma(\infty)=z$ and its boundary $\operatorname{HS}(p, z)$ is called a horosphere with center $z$. In this case, we define the Busemann function by

$$
\Phi_{\gamma}(p)=\lim _{t \rightarrow \infty}(d(p, \gamma(t))-t)
$$

for the geodesic $\gamma$. Then, for the unit speed geodesic ray

$$
\gamma(0)=p, \quad \gamma(\infty)=z \in N(\infty),
$$


the following results are well known (see [4], [14]):

$$
\operatorname{HB}(p, z)=\Phi_{\gamma}^{-1}(-\infty, 0), \quad \operatorname{HS}(p, z)=\Phi_{\gamma}^{-1}(0) .
$$

For a unit speed geodesic ray $\gamma$, if

$$
\gamma(0)=x \in M, \quad \gamma(\infty)=z \in N(\infty)
$$

and

$$
\gamma^{\prime}(0) \in T_{x} M^{\perp},
$$

then the horosphere $\operatorname{HS}(x, z)$ is called the tangent horosphere $\operatorname{HS}(x)$ to $M$ at $x \in M$. A hypersurface $M$ in $N$ is said to be convex at a point $x \in M$ if the tangent horosphere at $x$ does not seperate a neighborhood of $x$ into two parts. Moreover, if $x$ is the only point of a neighborhood which lies on $\operatorname{HS}(x)$, then $M$ is said to be strictly convex at $x$. A convex set by horoballs in a Hadamard manifold $N$ is a subset $D$ in $N$ with boundary $\partial D$ satisfying that, for every $x \in \partial D$ there is a horosphere of $N$ through $x$ such that $D$ is locally contained in the horoball of $N$ bounded by the horosphere. This horosphere is called a supporting horosphere of $D$. For Hadamard manifolds $N$ with sectional curvature $K$ satisfying

$$
-k_{1}^{2} \geq K \geq-k_{2}^{2}, \quad k_{1}, k_{2}>0,
$$

if $\mathrm{HS}$ is a horosphere, at each point of HS where the normal curvature $k$ is well defined, it satisfies

$$
k_{1} \leq k \leq k_{2} .
$$

At other points in HS, the lower and upper normal curvatures $\underline{k}$ and $\bar{k}$ are well defined, and they still satisfy

$$
k_{1} \leq \underline{k} \leq \bar{k} \leq k_{2} .
$$

An orientable regular hypersurface $M$ of a Hadamard manifold $N$ is said to be $\lambda$-convex if, for a suitably chosen unit normal vector $\zeta$, the normal curvature $k \geq \lambda$. A domain $D$ in $N$ is $\lambda$-convex if, for every point $x \in \partial D$, there is a regular $\lambda$-convex hypersurface $M$ through $x$ leaving a neighborhood of $x$ in the convex side (the side where the unit normal vector points) of $M$. If $\partial D$ is regular, then it is a regular $\lambda$-convex hypersurface. We will also recall that if $D$ is convex in $N$, then $\partial D$ is a topological embedded hypersurface which is smooth except for a set of zero measure.

Moreover, we have the following result: 
THEOREM 2.1 ([1] and [6]). If $M$ is a compact orientable, locally convex and immersed hypersurface of a Hadamard manifold $N$, then $M$ is embedded, homeomorphic to sphere, and is the boundary of a convex set $D$.

By the definition and (3) we easily have

Lemma 2.2. $M$ is convex (resp. strictly convex) at $x \in M$ if and only if the Busemann function $\Phi_{\gamma}(y)$ takes non-negative value in a neighborhood $V$ of $x$ in $M$ (resp. moreover $\Phi_{\gamma}(y)>0$ for all $y \in V, y \neq x$ ).

Now let $S_{p}^{n}$ be the unit sphere in $T_{p} N$ at a point $p \in N$. For $s \in S_{p}^{n}$ let $\gamma_{s}: \mathrm{R} \rightarrow N$ be the unit speed geodesic ray with $\gamma_{s}(0)=p, \gamma_{s}^{\prime}(0)=s$. Then, the mapping $\Psi_{p}: S_{p}^{n} \rightarrow N(\infty), \Psi_{p}(s):=\gamma_{s}(\infty)$ is a homeomorphism [12]. Supposing that $M$ is orientable in $N$, we may take a unique outward unit normal vector $\zeta_{x} \in T_{x} M^{\perp}$ and so we can define the mapping $j: M \rightarrow N(\infty), j(x):=$ $x(\infty)=\gamma(\infty)$, where $\gamma$ is a unit speed geodesic ray emanating from $x$ satisfying $\gamma^{\prime}(0) \in T_{x} M^{\perp}$. Thus, when $M$ is orientable, denoting $S^{n}=S_{q}^{n}$ for a fixed point $q \in N$, we get the mapping $G=\Psi_{q}^{-1} \circ j: M \rightarrow S^{n}$, which may be called the Gauss map. Now, the unit normal vector field $\zeta$ on $M$ may be considered a differentiable mapping which corresponds at a point $x \in M$ to the unit normal vector $\zeta_{x}$ to $M$ at $x$. Therefore, the mapping $\Psi_{q}^{-1} \circ \Psi_{x}: S_{x}^{n} \rightarrow S^{n}$ is clearly a homeomorphism. By these mappings, the Gauss map $G$ may be considered as the composed mapping $G(x)=\left(\Psi_{q}^{-1} \circ \Psi_{x} \circ \zeta\right)(x): M \rightarrow S^{n}$, for $\forall x \in M$. Since $M$ is compact orientable immersed hypersurface of the Hadamard manifold $N, M$ lies in some metric ball $B$ and $K<0$ implies that $\exp _{x}$ is a diffeomorphism onto $N$, for any $x \in N$, and metric balls are convex. So, for any $y \in M$, the geodesic ray $\gamma_{y}$ in $N$ with initial direction $\zeta(y)$ strikes $\partial B$ once and transversely at a point of $\partial B$. If $M$ has no focal points on $\gamma_{y}$, then the map $M \rightarrow \partial B$ is a diffeomorphism, since $\partial B$ is diffeomorphic to $S^{n}$. Therefore, we have the following theorem by utilizing the Rauch comparision theorem.

THEOREM 2.3 ([1]). If $M$ is a compact orientable locally convex immersed hypersurface of a Hadamard manifold $N$ and if the sectional curvatures of $N$ satisfy $K \leq-k \leq 0$ and the continuous unit normal $\zeta$ of $M$ may be chosen so that the eigenvalues of the second fundamental form $\sigma$ satisfy $\lambda \geq-\sqrt{k}$, then $M$ is embedded, diffeomorphic to the sphere $S^{n}$, and is the boundary of a convex set.

Let $h:[0, l] \times I \rightarrow N$ be a differentiable map. A differentiable vector field along $h$ is a differentiable map $V:[0, l] \times I \rightarrow N$ such that $V(s, \epsilon) \in T_{h(s, \epsilon)} N$ for each $(s, \epsilon) \in[0, l] \times I$. In what follows the following proposition will be needed. 
Proposition 2.4 ([10]). A regular parametrized curve $\gamma:[0, l] \rightarrow N$, where the parameter $s \in[0, l]$ is the arc length of $\gamma$, is a geodesic curve if and only if for every proper variation $h:[0, l] \times I \rightarrow N$ of $\gamma, L^{\prime}(0)=0$ where $L$ is the arc length of the variational curve $h_{\epsilon}$ for $\epsilon \in I$.

By theorem 2.1 together with above, we easily see that the mapping $S^{n} \rightarrow$ $N(\infty)$ is a diffeomorphism.

\section{Proof of the Theorem}

Now we will prove our assertions in the theorem. In our proof geodesic variations will be the basic tool. Let us assume that the second fundamental form $\sigma$ of $M$ be definite on a neighborhood $U$ of a point $x$ of $M$. Then, since the second fundamental form $\sigma$ will be non-degenerate on $U$, so definite on $U$ for a suitably chosen unit normal $\zeta$, it is clear that the eigenvalues $\lambda$ of the second fundamental form $\sigma$ satisfy $\lambda>-\sqrt{k}$. Now, before continuing the proof we will prove

LEMMA 3.1. Let $M$ be an $n$-dimensional compact submanifold in an $(n+m)$ dimensional Hadamard manifold $N$. Then there exists a point $x_{0} \in M$ such that $\sigma$ is definite at $x_{0}$.

Proof. Let us take a point $y_{0}$ of $N$ and fix it. Since $M$ is compact, there is a point $x_{0}$ of $M$ so that the distance function $\operatorname{dist}\left(y_{0}, y\right)=d(y)$ takes maximum value at this point. After that, we may take a unit speed geodesic ray $\gamma:[0, l] \rightarrow N, \gamma(0)=x_{0}, \gamma^{\prime}(0)=\zeta_{0} \in T_{x_{0}} M^{\perp}$, and a tangent vector $X_{0} \in T_{x_{0}} M$. Then, we consider a curve $y(\epsilon)$ such that $y(0)=y_{0}, y^{\prime}(0)=X_{0}$ and $y(\epsilon) \in M$ for $\epsilon \in I$, where $I$ is an open interval around the origin of $\mathrm{R}$. Thus, we can consider a variation $h$ of $\gamma$, that is, a differentiable mapping $h:[0, l] \times I \rightarrow N$ such that $h(s, 0)=\gamma(s)$ for each $s \in[0, l]$ and $h(l, \epsilon)=$ $\gamma(l)$ for each $\epsilon \in I$ and each variational curve $h_{\epsilon}(s)=h(s, \epsilon)$ is a geodesic curve from $y(\epsilon)$ to $\gamma(l)$. Let $L(\epsilon)=L\left(h_{\epsilon}\right)$ be the arc length of a variational curve $h_{\epsilon}$. As is well known, the function $L$ is differentiable, and so we have the variational equations of $L$ in a neighborhood of zero as the following:

$$
\begin{aligned}
L^{\prime}(0)= & {[\tilde{g}(X, \zeta)(s, 0)]_{0}^{l} } \\
L^{\prime \prime}(0)= & \int_{0}^{l}\left[\tilde{g}\left(\tilde{\nabla}_{\partial_{1}} \tilde{X}, \tilde{\nabla}_{\partial_{1}} \tilde{X}\right)-\tilde{g}(\tilde{R}(X, \zeta) \zeta)(s, 0)\right] d s \\
& +\left[\tilde{g}\left(\tilde{\nabla}_{\partial_{2}} X, \zeta\right)(s, 0)_{0}^{l}\right],
\end{aligned}
$$

where $\tilde{R}$ is the Riemannian curvature tensor of $N, \zeta=h_{*} \partial_{1}, X=h_{*} \partial_{2}$, and $\tilde{X}=X-\tilde{g}(X, \zeta) \zeta$. For this variation, it is well known that $X_{0}=X(0,0)=$ 
$h_{*}\left(\partial_{2}(0,0)\right)$ and $X(s, 0)=h_{*}\left(\partial_{2}(s, 0)\right)$ is a Jacobi field along $\gamma$, that is, $X^{\prime \prime}(0)+\tilde{R}(X(s, 0), \zeta(s, 0)) \zeta(s, 0)=0$. Since $\zeta(s, 0)=\gamma^{\prime}(s), \tilde{X}(s, 0)=$ $X(s, 0), X(l, \epsilon)=0$ and $\tilde{g}(X(0,0), \zeta(0,0))=0$, from Proposition 2.4 we have $L^{\prime}(0)=0$ and

$$
\begin{aligned}
L^{\prime \prime} & =\int_{0}^{l}\left[\tilde{g}\left(X^{\prime}, X^{\prime}\right)+\tilde{g}\left(X^{\prime \prime}, X\right)\right](s, 0) d s-\tilde{g}\left(\sigma\left(X_{0}, X_{0}\right), \zeta_{0}\right) \\
& =\tilde{g}\left(X^{\prime}, X\right)(l, 0)-\tilde{g}\left(X^{\prime}, X\right)(0,0)-\tilde{g}\left(\sigma\left(X_{0}, X_{0}\right), \zeta\right) \\
& =-\tilde{g}\left(\sigma\left(X_{0}, X_{0}\right), \zeta_{0}\right) .
\end{aligned}
$$

Since the curve $h_{0}=\gamma$ is the shortest geodesic segment among the all variational curves, we have $L^{\prime \prime}(0)>0$ and hence $\sigma\left(X_{0}, X_{0}\right) \neq 0$. Since $X_{0}$ is any tangent vector to $M$ at $\gamma(0)=x_{0}, \sigma$ is definite at $x_{0}$. Thus we have proved the lemma.

Now, let us prove that (i) implies (ii). Since the second fundamental form $\sigma$ is non-degenerate on $M$, it is clear that $K \neq 0$, and by the hypothesis $K \leq-k<0$ we get that all the eigenvalues of $\sigma$ are different from zero, that is all $\lambda \neq 0$. This proves (i) $\Rightarrow$ (ii).

Now, let us show that (ii) implies (i). If the conditions in (ii) hold, then $M$ is diffeomorphic to the sphere $S^{n}$ from Theorem 2.3. For Hadamard manifolds with sectional curvatures $K$ satisfying $-k_{1}^{2} \geq K \geq-k_{2}^{2}, k_{1}, k_{2}>0$, if HS is a horosphere, then at each point of HS where the normal curvature $k$ is well defined, it satisfies $k_{1} \leq k \leq k_{2}$. At each point in HS the lower and upper normal curvatures $\underline{k}$ and $\bar{k}$ are well defined, and they still satisfy $k_{1} \leq \underline{k} \leq \bar{k} \leq k_{2}$. Since (HS $\cap M) \subset N$, this implies that all sectional curvatures at every point of $M$ are different from zero and so $\sigma$ is non-degenerate everywhere on $M$. Since $M$ is connected and, from Lemma 3.1, $\sigma$ is definite at $x_{0}$, then $\sigma$ is definite everywhere on $M$. Thus, we completed the proof of (ii) $\Rightarrow$ (i).

Now, for a suitably chosen continuous unit normal $\zeta$ of $M$ the second fundamental form $\sigma$ of $M$ is definite everywhere on $M$. Since $M$ is compact, it lies in some metric ball $\Omega$. Then, we have a diffeomorphism $f: M \rightarrow \partial \Omega$. If we define $P: M \times(-\epsilon, \infty) \rightarrow N, P(y(t), t)=\exp \zeta(y(t)), y(t) \in M$, then we have a geodesic ray $\gamma_{y(t)}$ by fixing $y(t)$. Now, if $\gamma^{\prime}(0)=\zeta_{0} \in T_{y(t)} M^{\perp}$, then any field $X$ on $M$ is orthogonal to $\zeta_{0}$ along $\gamma$ and satisfies $X_{y(t)}(0)=$ $\gamma_{* 0}(X(y(t)))$. So along $\gamma, X$ is a Jacobi field, and therefore $g(X, X)$ is convex and so $2 g\left(\sigma_{\zeta} X, X\right)=\zeta . g(X, X)$ is non-decreasing. Thus, by a theorem of Bishop [5], at each point of $M, \gamma$ is locally convex away from $\zeta(y(t), 0)$. By the hypothesis of connectedness we get $M$ is locally convex. This completes the proof of (i) $\Rightarrow$ (iii).

By the theorem of Borisenko [6], [7], $M$ is a convex hypersurface. So, to 
complete the proof of theorem we only need to prove that one of the three conditions implies that $M$ is strictly convex.

Let us take a point $x_{0} \in M$ and let $\gamma$ be a unit speed geodesic ray emanating from $x_{0}$ with $\gamma^{\prime}(0)=\zeta_{x_{0}}$, which is a outward unit normal vector to $M$ at $x_{0}$. Then, we get the Busemann function $\Phi_{\gamma}$ for the geodesic $\gamma$, and so the horosphere $\operatorname{HS}\left(x_{0}\right)=\Phi_{\gamma}^{-1}(0)$ at $x_{0}$. We would like to show that the point $x_{0}$ is an isolated point in $M \cap \mathrm{HS}\left(x_{0}\right)$ and $M-\left\{x_{0}\right\}$ is in the region $\Phi_{\gamma}^{-1}(0, \infty)$. Suppose that we have another point $x \in M$ in the horoball $\operatorname{HB}\left(x_{0}\right)=\Phi_{\gamma}^{-1}(-\infty, 0]$. Then, we have a subset $U$ of $M$ in $\operatorname{HB}\left(x_{0}\right)=\Phi_{\gamma}^{-1}(-\infty, 0]$. The function $\Phi_{\gamma}$ attains its minimum at a point $x_{1} \in U$ on $M$. Then the horosphere $\operatorname{HS}\left(x_{1}\right)$ has the same center $\gamma(\infty)$ as the one of $\operatorname{HS}\left(x_{0}\right)$, and so we have $G\left(x_{1}\right)=G\left(x_{0}\right)$, which implies $x_{1}=x_{0}$, because the Gauss map $G$ is a homeomorphism. Thus, $M-\left\{x_{0}\right\}$ lies in the region $\Phi_{\gamma}>0$, that is, $x_{0}$ is an isolated point of $M \cap \operatorname{HS}\left(x_{0}\right)$ and $M-\left\{x_{0}\right\}$ is in the region $\Phi_{\gamma}^{-1}(0, \infty)$. Hence we have proved that $M$ is a strictly convex hypersurface of Hadamard manifold $N$.

\section{REFERENCES}

1. Alexander, S. B., Locally convex hypersurfaces of negatively curved spaces, Proc. Amer. Math. Soc. 64 (1977), 321-325.

2. Alexandrov, A. D., Complete convex surfaces in Lobachevsky space, Izv. Akad. Nauk SSSR Ser. Mat. 2 (1945), 113-120.

3. Alexandrov, A. D., Intrinsic Geometry of Convex Surfaces (in Russian), German Translation: Die Innere Geometrie der konvexen Flächen, Akad. Verl., Berlin (1955).

4. Ballmann, W., Gromov, M., and Schroeder, V., Manifolds of non-positive Curvature, Birkhauser, Boston, Basel, Stuttgart (1985).

5. Bishop, R. L., Infinitesimal convexity implies local convexity, Indiana Univ. Math. J. 24 (1974/75), 169-172.

6. Borisenko, A. A., On locally convex hypersurfaces in Hadamard manifolds, Math. Notes 67 (2000), 425-432.

7. Borisenko, A. A., and Miquel, V., Comparison theorems on convex hypersurfaces in Hadamard manifolds, Ann. Global Anal. Geom. 21 (2002), 191-202.

8. Burago, D., Burago, Y., and Ivanov, S., A Course in Metric Geometry, Grad. Stud. Math. Vol. 33 (2001).

9. Burago, Yu A., Zalgaller, V. A., Convex sets in Riemannian manifolds, Uspekhi Mat. Nauk. 2(32) (1977), 5-51.

10. do Carmo, M. P., Differential Geometry of Curves and Surfaces, Prentice Hall Inc., New Jersey (1976).

11. Chern, S. S., and Lashof, R. K., On the total curvature of immersed manifolds, Amer. J. Math. 79 (1957), 306-318.

12. Chern, S. S., and Lashof, R. K., On the total curvature of immersed manifolds, Michigan Math. J. 5 (1958), 5-12.

13. Hadamard, J., Les surfaces à courbures opposées et leurs linges géodésiquese, J. Math. Pures Appl. 4 (1898), 27-73. 
14. Heintze, E., and Hopf, H. I., Geometry of horosphere, J. Differential Geom. 12 (1977), 481491.

\author{
DEPARTMENT OF MATHEMATICS \\ FACULTY OF SCIENCE \\ ISTANBUL UNIVERSITY \\ 34459 VEZNECILER \\ ISTANBUL \\ TURKEY \\ E-mail: erdoganm@istanbul.edu.tr
}

